\title{
El papel de la didáctica en los procesos de enseñanza y aprendizaje universitarios
}

Recibido: $\quad 28$ de febrero, 2020.

Aceptado: $\quad 27$ de abril, 2020.

Por: Dr. Wilmer Casasola Rivera ${ }^{1}$, Instituto Tecnológico de Costa Rica, Costa Rica, ORCID: 0000-0002-0927-5049

\section{Resumen}

El artículo se desprende de un capítulo de tesis doctoral, pero añade un apartado diferente para contextualizar el tema de la didáctica universitaria. El trabajo se enfoca en exponer un cuerpo teórico de conocimiento sobre el papel de la didáctica universitaria en los procesos de enseñanza y aprendizaje. Describe un marco de conocimiento básico pero sustentable sobre el tema que procura ser una invitación a la investigación, análisis, reflexión y aplicabilidad de la didáctica al ámbito formativo, principalmente universitario. El trabajo se aborda desde un enfoque cualitativo y una técnica de investigación documental.

\section{Abstract}

\section{The role of didactics in the university teaching and learning processes}

This study stems from the findings in a doctoral thesis chapter, but adds a different section to address the topic of university teaching. This article focuses on outlining a theoretical framework of knowledge about the role of university didactics in the teaching and learning processes. It sets forth a basic but sustainable framework of knowledge on the subject, aimed at fostering research, analysis, reflection, and the applicability of didactics to education, mainly in the context of higher education. The study resorts to a qualitative approach and a documentary research technique.

1 El profesor Wilmer Casasola Rivera es Doctor en Educación, graduado en la Universidad de Baja California, México. Labora en la Escuela de Ciencias Sociales del Instituto Tecnológico de Costa Rica, Costa Rica, como docente e investigador. Contacto: wcasasola@itcr.ac.cr.

\section{PALABRAS CLAVE:}

Didáctica, didáctica universitaria, estrategias

didácticas, enseñanza, enseñanza universitaria, aprendizaje, docencia

universitaria, universidad.

KEY WORDS:

Didactics, university didactics, didactic strategies, teaching, university teaching, learning, university learning, university. 


\section{INTRODUCCIÓN}

El artículo se desprende de un capítulo de tesis doctoral, sobre estrategias didáctica y habilidades metacognitivas en docentes y en estudiantes universitarios, presentada en México, en el año 2018. No obstante, se añade un apartado diferente $y$, por tanto, exclusivo para este artículo, con el fin de contextualizar el tema de la didáctica universitaria. La tesis doctoral, como tal, no ha sido publicada digitalmente.

Este artículo aborda el tema de la didáctica en el contexto universitario. No se trata de un estudio particular, sino de una reflexión general sobre la importancia que tiene esta disciplina en los procesos de enseñanza y aprendizaje. En consecuencia, su objetivo central se enfoca en exponer un cuerpo teórico de conocimientos sobre el papel de la didáctica universitaria en los procesos de enseñanza y aprendizaje.

Construir una perspectiva teórica sobre la importancia de la didáctica en el proceso de enseñanza-aprendizaje es una tarea compleja. Cuando se aprecia la abundante literatura sobre didáctica en los estantes de las bibliotecas, la cantidad de libros en las librerías y la portentosa producción de libros digitales sobre este tema, se llega a la conclusión de que la tarea es abrumadora. Mucho se dice sobre didáctica, tanto aplicada a los primeros niveles escolares, como en los niveles más elevados de la formación académica.

El trabajo ofrece un aporte al estudio y la reflexión sobre la didáctica universitaria. Describe un marco de conocimiento básico, pero sustentable, sobre el tema que procura ser una invitación para la investigación, el análisis, la reflexión y la aplicabilidad de la didáctica al ámbito formativo, cualquiera sea su nivel.

En cuanto a cuestiones metodológicas, este trabajo parte de un enfoque cualitativo-documental. Por una parte, no ofrece un apartado con discusión de resultados, como usualmente lo demanda el paradigma cuantitativo, y del que también suele ser partícipe la escritura cualitativa. La recolección y análisis de la información ocurren prácticamente en paralelo. Por otra parte, con base en el desarrollo de una investigación documental, la fase de análisis de datos se presenta como un proceso que busca dar sentido a los documentos a partir de la existencia de la información recogida (Hernández-Sampieri, 2014; Rodríguez-Gómez \& Valldeoriola-Roquet, 2009). De acuerdo con lo anterior, el artículo se enfoca en desarrollar un marco de referencia teórico sobre el tema central. En la sección correspondiente a las conclusiones, se realiza una síntesis de las principales ideas desarrolladas, así como, algunas reflexiones sobre el tema tratado.

En concordancia con lo anterior, es importante tener en cuenta que este artículo no busca resolver ni analizar formalmente un problema de investigación. El planteamiento de alguna pregunta podría provocar un cambio en el enfoque metodológico documental y su objetivo consiste en mostrar una perspectiva o marco teórico general sobre la didáctica universitaria.

El artículo se desarrolla en dos apartados centrales. El primer apartado expone los fundamentos teóricos del tema a tratar. El segundo apartado es una reflexión ensayística sobre la importancia de la investigación e innovación en la docencia universitaria.

Finalmente, el uso de un género determinado no es excluyente, sino que su uso obedece a una economía de lenguaje y una redacción más fluida. En ningún momento, cuando se habla de docente o profesor, se excluye la labor de la docente o la profesora en el contexto universitario o de otros niveles de formación.

\section{EL PAPEL DE LA DIDÁCTICA EN LA ENSEÑANZA UNIVERSITARIA}

En este apartado se desarrollan tres temas como parte del fundamento teórico del artículo. En el primer tema, se discute la importancia de la didáctica en el proceso de enseñanza-aprendizaje; en el segundo, se explica el concepto de estrategias didácticas; $y$, en el último, se expone el papel de la didáctica universitaria y su importancia. 
El papel de la didáctica en los procesos de enseñanza y aprendizaje universitarios

\section{LA DIDÁCTICA EN EL PROCESO DE ENSEÑANZA-APRENDIZAJE}

Previo al desarrollo puntual de este este apartado, es importante realizar algunas acotaciones etimológicas e históricas del concepto didáctica. Esto permite demarcar aún más la comprensión de esta disciplina y su importancia en el campo de la educación.

La palabra didáctica proviene del griego didaskein. Significa enseñar, instruir, explicar, hacer, saber, demostrar. También, la etimología griega pasó al latín, en la voz discere y docere que significan, respectivamente, aprender y enseñar. El uso del término didáctica en la actualidad conserva el significado original del griego y latín (Escribano-González, 2004).

Es en el año 1629, en el libro Principales Aforismos Didácticos, del educador alemán Wolfgang Ratke (1571-1635), cuando se emplea por primera vez la palabra didáctica. Sin embargo, el término se consagra en el siglo XVII con el teólogo y filósofo Jan Komensky, más conocido como Comenio (1592-1670), quien publica, en 1657, su obra Didáctica Magna, lo cual otorga de manera definitiva el carácter pedagógico y sienta los fundamentos de la didáctica como disciplina metodológica para organizar la enseñanza. Siglos más tarde (XIX), otros pensadores darían a la didáctica las bases de un conocimiento centrado en la organización de la enseñanza y del aprendizaje de los estudiantes, como Herbert y Willman (Escribano-González, 2004; Torres-Maldonado y Girón, 2009; García, 2015).

La didáctica, por su parte, se divide en didáctica general y didáctica especial. La didáctica general se encarga del estudio de las bases y fundamentos que soporta esta ciencia. Parte de un planteamiento integral de los principales elementos que concurren en el acto didáctico, como el conjunto de principios, técnicas, modelos, estrategias, entre otros, generalizables a contextos y niveles escolares diferentes. La didáctica especial, por su parte, se orienta a los diferentes contenidos curriculares de un área de conocimiento concreta; es decir, a campos de conocimientos que requieren de una didáctica particular para lograr que el proceso de enseñanza y aprendizaje sea el más adecuado (Escribano-González, 2004).
La didáctica general permite una comprensión integral de los recursos pedagógicos fundamentales en el proceso de planificación de la enseñanza y del aprendizaje, y la didáctica especial puntualiza en la creación de estrategias específicas para optimizar este proceso. Es el campo de la especialización didáctica donde se delimita el nivel educativo al que tiene que responder, con diferentes recursos didácticos, para garantizar un adecuado proceso tanto en la enseñanza como en el aprendizaje.

Una de las primeras consideraciones que debe tener un docente en los procesos de planificación didáctica es contar con el conocimiento de los cambios que se producen en los estudiantes según sus edades. Esto con el fin de enfocar, dirigir y dinamizar los procesos de enseñanza y aprendizaje, para alcanzar un mayor aprovechamiento durante una sesión de trabajo en el salón de clases. (Cervera, 2010). Si bien es cierto que en la formación universitaria existe cierta homogeneidad en la edad, también es cierto que la edad de ingreso a la universidad suele variar. No obstante, es importante tener en cuenta que la edad no significa uniformidad en el nivel de madurez de los estudiantes, tanto en la forma de asumir los estudios universitarios como las metas de crecimiento profesional. Es por esta razón que la planificación didáctica, incluso en la universidad, es una necesidad incuestionable. Todo docente tiene que prestar atención a esta variable.

Cuando se dan este tipo de eventos, es necesario considerar la importancia de la planificación didáctica. El planeamiento didáctico es uno de los momentos fundamentales en los procesos de enseñanza y aprendizaje. Según Rojas-Vargas (2016), el planeamiento didáctico debe estar basado en un paradigma educativo y en un modelo pedagógico. Esto permitiría contar con una guía teórica para orientar la labor docente y el trabajo de planificación didáctica. Sin embargo, no solo el planeamiento didáctico debe estar basado en un paradigma educativo y en un modelo pedagógico, sino también en un paradigma de la investigación continua de los procesos didácticos para garantizar un óptimo aprendizaje. La investigación didáctica es de suma importancia porque ningún aprendizaje está determinado, así como, ningún 
método de enseñanza garantiza verdaderamente un óptimo aprendizaje en el estudiante.

También es importante tomar en cuenta una realidad insoslayable de la planificación didáctica: la generalización de un método didáctico es difícil. El establecimiento de un modelo didáctico que pueda adaptarse de forma general a cualquier grupo de estudiantes es problemático. Existe cierto riesgo al pretender establecer modelos pedagógicos estables a partir de la implementación de métodos didácticos generalizados. Varios factores condicionan la pretensión de establecer un modelo pedagógico general, entre ellos: las características físicas y psicológicas, las relaciones interpersonales del grupo, la relación entre el docente y el grupo de estudiantes en general. Todos estos son factores que condicionan la planificación didáctica. No obstante, cuando existe evidencia generalizada de que algún modelo o estrategia didáctica funciona, se deben adaptar (Cervera, 2010).

En relación con lo anterior, todo modelo didáctico que se quiera implementar debe contar con los recursos indispensables que faciliten su aplicación, con el modelo de evaluación de los resultados y con el establecimiento de estrategias de modificación o adaptación, cuando se da una desviación de los objetivos trazados. La evaluación no debe ser sancionadora, sino formativa, motivadora y orientadora. La evaluación tiene que convertirse en una actividad de conocimiento y la corrección en un acto de aprendizaje (Cervera, 2010; Ortega-Alfaro, 2017).

Los modelos didácticos tienen como meta intervenir en los procesos pedagógicos con el fin de lograr optimizar las competencias en los estudiantes y, a la vez, facilitar la labor docente (Cervera, 2010). Para esto, se requiere establecer una estructura organizada con los elementos de control necesarios que permitan readaptar el diseño o la planificación didáctica. Si bien es cierto que elegir y poner en práctica una estrategia didáctica no está exenta de dificultades, permite al docente contar con una guía para evaluar el aprendizaje. Los procesos de planificación de los aprendizajes, o bien la planificación didáctica, requieren de una verdadera investigación. De acuerdo con CantúLozano (2016), la investigación se debe realizar con el propósito de efectuar la selección de contenidos y actividades de aprendizaje, para dar prioridad a lo que es significativo. El docente debe partir de lo conocido a lo desconocido, para llevar al alumno de la comprensión a la aplicación, con el fin de que aprenda haciendo y aprenda a aprender.

La planificación didáctica permite identificar muchas dificultades en los procesos de aprendizaje y, también, ha logrado reorientar los procesos de aprendizaje de manera más eficaz. Sin embargo, debe existir cierta disposición en el profesorado para superar este obstáculo. El docente tiene que ajustarse a los cambios sociales derivados de las tecnologías de la información y la comunicación, y adaptarlos de manera novedosa a su actividad cotidiana. Para ello, la planificación didáctica tiene que orientarse necesariamente hacia la investigación, donde uno de sus objetivos centrales es la innovación de la docencia y el mejoramiento de las habilidades de aprendizajes de los estudiantes. Con este fin, se inicia la investigación didáctica como recurso para mejorar la experiencia de la enseñanza y del aprendizaje (Cervera, 2010; Medina \& Medina, 2014).

Desde luego que la investigación didáctica implica un abordaje metodológico riguroso. El planteamiento de un adecuado diseño de investigación didáctica implica un apropiado tratamiento de las observaciones y del análisis. El manejo o manipulación de variables se abordan desde un análisis estadístico y de las diversas formas de obtención y análisis de datos para contar con conclusiones derivadas del análisis realizado (Escribano-González, 2004). Este trabajo no se ocupa del procedimiento metodológico para la investigación didáctica, sino que hace referencia a la importancia que tiene la investigación didáctica en la innovación de los procesos de enseñanza y aprendizaje.

La investigación didáctica es una base para enfocar los procesos de enseñanza y aprendizaje. Este tipo de investigación genera conocimientos que permiten comprender y actuar en los procesos de enseñanzaaprendizaje a través de metodologías que logran fundamentar y promover la didáctica, la cual orienta las acciones de enseñanza y aprendizaje en el ámbito 
educativo. Idealmente, la didáctica busca continuamente nuevos planteamientos que permitan actuar y enriquecer enfoques teóricos, modelos y prácticas educativas que mejoren la experiencia de aprendizaje en las aulas; así como, la satisfacción de estudiantes y docentes en la tarea escolar, y la adaptación y el desarrollo integral de las instituciones educativas (Medina \& Medina, 2014).

Los procesos de investigación didáctica para el mejoramiento de la calidad de la enseñanza y el aprendizaje implican, de forma directa o indirecta, la innovación educativa, específicamente, los proyectos de innovación didáctica. Tales proyectos son estrategias de planificación y acción profesional que permiten a los docentes controlar su práctica profesional educativa a través de una metodología de indagación-solución que implica tres fases elementales: planeación, implementación y evaluación. El punto de partida para la innovación didáctica lo constituye la elección de un tema que inquiete al docente y que esté relacionado con su práctica profesional, denominado Preocupación Temática, el cual hace referencia al ámbito empírico de acción de la práctica profesional del docente en donde puede innovar. Planificar una estrategia didáctica, implementar la estrategia, y evaluar los resultados de la estrategia son parte de esta metodología (Barraza-Macías, 2015).

La innovación didáctica, en el contexto de la investigación, no es un proceso de ensayo y error. Como se formuló antes, es un proceso metodológicamente planificado. Ahora bien, la didáctica, comprendida dentro del enfoque cognitivo del aprendizaje, busca desarrollar en los estudiantes habilidades para enfrentar la tarea de aprender de una forma autónoma. Innovar didácticamente dentro del contexto de la psicología cognitiva del aprendizaje significa facilitar al estudiante las herramientas necesarias que les permita potenciar y desarrollar habilidades metacognitivas para el aprendizaje.

Para este fin, como puntualiza Sebastiá-Alcaraz (2012), debe existir intencionalidad en la innovación, un fin previsto que busque mejorar, de forma eficaz (buscar mejores resultados) o eficiente (reducir los esfuerzos que conducen a un determinado resulta- do) el proceso educativo. Entonces, la innovación se produce cuando se incorpora algo que anteriormente no estaba presente en la esencia de lo innovado y que añade diferencias sustanciales al proceso. De ahí que desarrollar las habilidades de aprendizaje en los estudiantes de forma autónoma tiene que ver con innovación didáctica, cuando se revisa si se quiere mejorar la eficacia o la eficiencia en el proceso de aprendizaje.

Uno de los objetivos del enfoque cognitivo del aprendizaje es despertar en el estudiante la habilidad de procesar y dar significado a la información, y generar de forma autónoma sus propias estrategias de aprendizaje. En las personas, las estructuras cognitivas representan de forma organizada las experiencias previas, pues funcionan como esquemas activos para filtrar, codificar, categorizar y evaluar la información que reciben de alguna experiencia relevante de aprendizaje (Chadwick, 1999). Por tal motivo, el proceso de planificación didáctica, desde este enfoque, debe procurar despertar estas habilidades en cada estudiante. En este sentido, los enfoques cognitivos del aprendizaje invitan al docente a abandonar el tradicionalismo didáctico. Esto es el procedimiento rutinario en que el docente se limita a presentar y a proporcionar contenidos de forma magistral, lo cual facilita su labor, pero no la del estudiante, pues dificulta el camino hacia su aprendizaje autónomo.

Por el contrario, la búsqueda de alternativas didácticas es un compromiso cuando se quiere resultados que motiven y afecten positivamente el rendimiento académico, para lo cual se requiere de actividades que involucren al estudiante a asumir mayor autonomía en su aprendizaje (aprendizaje por descubrimiento). Esto implica contar con recursos apropiados, como material didáctico y aulas apropiadas.

En el contexto de la enseñanza universitaria, los docentes deben procurar que los estudiantes aprendan a conocer nuevos conceptos, y no tanto a memorizarlos. La diferencia cualitativa está en ensañar a aprender al estudiante. $Y$ este el papel que juega el profesor universitario: un facilitador del aprendizaje. El estudiante asume la responsabilidad de lograr un aprendizaje significativo a través de 
las diversas actividades didácticas que promueve el docente (Roldán, 2004; Cervera, 2010).

La pedagogía tradicional emplea la estrategia didáctica tradicional: el discurso magistral en el salón de clases o, en palabras sencillas: hablar y hablar. De acuerdo con Cervera (2010), el avance de las tecnologías en nuestra sociedad ha creado tanto la necesidad de desarrollar nuevas habilidades en nuestros alumnos, como la necesidad de adquirir nuevos métodos pedagógicos que abandonen las limitaciones que impone la pedagogía tradicional. Fundamentalmente, el carácter discursivo (magistral) que se ha empleado a lo largo de años y que resulta menos atractivo y motivador para el aprendizaje de los estudiantes.

El avance de las tecnologías de la información provoca un cambio en el paradigma conservador del aprendizaje. Los educadores universitarios deben ampliar su perspectiva de enseñanza y ser receptivos a las nuevas tecnologías educativas con el fin de favorecer la educación universitaria. Los cambios provocados por la globalización y el avance de la ciencia y de la tecnología, advierten la necesidad de realizar un giro favorable en los profesores y una modificación de políticas en las instituciones de educación superior para mejorar la educación universitaria. El docente de aula es cada día menos competente para su labor de transferir información a los estudiantes (Roldán, 2004).

Lo anterior evidencia que existen factores condicionantes, pero no determinantes en el proceso de implementación de estrategias didácticas, y, por tanto, las variables ambientales (los propios estudiantes, el lugar físico, el conocimiento del profesor) son factores que condicionan el éxito, pero no determinan el fracaso pedagógico.

\section{EL CONCEPTO ESTRATEGIAS DIDÁCTICAS}

El concepto estrategia se ha definido de muchas formas de acuerdo con los intereses o usos del concepto mismo. Las definiciones clásicas se ubican en el contexto empresarial e implican una planificación y acción deliberadas. Como no existe consenso sobre una definición aceptada, algunos prefieren reflexio- nar sobre qué trata la estrategia, en lugar de ofrecer una definición exacta. En este sentido, la estrategia no radica solamente en el análisis racional y analítico, en el que se encuentra una única respuesta correcta, si no que pueden interferir aspectos emocionales. La estrategia se concibe como una disciplina práctica que puede implicar un ejercicio teórico, pero cuya prueba final de validez se da en la práctica, en la acción (Tovstiga, 2012).

La estrategia expresa el proceso de ideas enfocadas en anticipar acciones para conseguir un objetivo o conjunto de objetivos. Se comprende como un sistema de planificación, de procedimientos organizados, formalizados y orientados a obtener una meta definida a través de un conjunto articulado de acciones. Se trata de herramientas del pensamiento que, a través de una serie de procedimientos, guían las acciones para alcanzar un objetivo o determinadas metas de aprendizaje (Montealegre-García, 2016; Sánchez-Romero, 2013).

No se puede hablar de estrategia cuando no hay metas hacia donde se orienten las acciones. Por tal motivo, la elección y diseño detallado de procedimientos y técnicas son responsabilidad del docente. La estrategia debe estar fundamentada en un método. En el campo propiamente educativo, el concepto estrategia se aplica para denotar el proceso sistemático de enseñanza y aprendizaje. En este sentido, algunos hablan de estrategias de enseñanza y otros de estrategias didácticas. Algunas veces, incluso, se habla de estrategias didácticas sin definir el concepto mismo de estrategia didáctica.

Para Pimienta-Prieto (2012), las estrategias de enseñanza y aprendizaje son instrumentos que facilitan al docente la implementación y desarrollo de competencias en los estudiantes. Para SánchezRomero (2013), la estrategia se concibe como una herramienta didáctica que permite dirigir un proceso para conseguir una finalidad. La estrategia es un procedimiento que posibilita desarrollar operaciones, actividades y planes para facilitar, adquirir y lograr un proceso de intervención de aprendizaje en el campo educativo. Por su parte, Montealegre-García (2016) señala que las estrategias didácticas también suelen 
denominarse métodos de enseñanza. Propiamente, son procedimientos o instrucciones ordenadas que facilitan la adquisición de experiencias de aprendizaje en los estudiantes.

Diferentes autores han clasificado las estrategias didácticas según el propósito de enseñanza que se quiere alcanzar. De acuerdo con Sánchez-Romero (2013), esta clasificación se puede dividir en cuatro tipos de estrategias didácticas: 1) Estrategias didácticas enfocadas en los procedimientos de aprendizaje; 2) Estrategias didácticas enfocadas en el proceso; 3) Estrategias didácticas enfocadas al comportamiento; 4) Estrategias didácticas enfocadas al contexto.

También, cabe agregar a esta clasificación las estrategias didácticas enfocadas en la enseñanza. La didáctica involucra las estrategias de enseñanza y de aprendizaje conjuntamente. En este sentido, las estrategias de enseñanza se refieren al diseño, programación, elaboración y formulación de contenidos de aprendizaje de forma verbal o escrita. Mientras que las estrategias de aprendizaje se refieren al diseño de estrategias que implementa el docente a través de la organización de la clase para que los alumnos aprendan a aprender. De tal forma que el diseño didáctico estimule en los estudiantes la observación, el análisis, la expresión de opiniones, la formulación de hipótesis, la propuesta de soluciones y el descubrimiento de conocimientos por sí mismos (Montealegre-García, 2016).

La pregunta ante el tema de las estrategias didácticas es cómo aplicarlas. Sánchez-Romero (2013) sugiere el cuestionamiento clásico: autorreflexión y planificación. Esto supone contar con una visión anticipadora de la acción que permita estructurar y evaluar el proceso de intervención didáctica y la toma de decisiones durante el proceso. Por tal motivo, la aplicabilidad de estrategias didácticas requiere atender la generalidad del contexto y la particularidad destinataria. Esto porque el contexto está en continuo cambio y se debe considerar la adaptación a este cambio.

\section{LA DIDÁCTICA UNIVERSITARIA}

Uno de los principales centros de interés de la investigación educativa actual lo constituye tanto la calidad de los procesos de enseñanza-aprendizaje, como la calidad profesional de los docentes (PrietoNavarro, 2007). Para este fin, la investigación e innovación didáctica son fundamentales en el quehacer de la docencia universitaria.

De acuerdo con Prieto-Navarro (2007), mejorar la docencia universitaria puede darse no solo a través de la actuación directa del profesorado en el aula, sino también a través de procesos de observación, de reflexión y de análisis de los profesores sobre su práctica docente y sobre sus creencias pedagógicas, quienes pueden ejercer una influencia indirecta en el aprendizaje de los alumnos. La búsqueda de la calidad en la docencia universitaria persigue la eficacia del aprendizaje

Se podría pensar que, en el contexto universitario, la didáctica ocupa un lugar preferencial, por tratarse del nivel de educación donde se privilegia la investigación y el conocimiento. Sin embargo, parece que no es así. A la universidad asisten profesionales a impartir lecciones de acuerdo con su formación particular, no necesariamente docentes.

De acuerdo con Zabalza-Beraza (2005), la identidad se vincula al campo científico al que pertenece el profesional, no necesariamente al de docente. La enseñanza universitaria constituye un espacio con escasa identidad profesional, pese a que el docente se declare profesor o docente universitario, no se ha preparado realmente para ser docente. "No todos los profesores universitarios piensan que la docencia sea algo realmente fundamental para la formación universitaria" (pp. 64-69). Muchos docentes consideran que a enseñar se aprende enseñando, que es una cuestión artística y depende de las cualidades particulares de cada docente.

Si bien es cierto que esta afirmación contiene un aspecto negativo, es una realidad que muchos hemos vivido, desde la formación de grado hasta posgrado $y$, por tal motivo, se puede considerar de vital importancia construir conocimiento didáctico para enfrentar con éxito el proceso de enseñanza con los estudiantes. En todo proceso de formación, el docente cumple un rol de facilitador de conocimiento, de destrezas, de habilidades. El profesor universitario es 
un facilitador del aprendizaje, cuyo objetivo es enfocarse en generar diferentes estrategias o actividades didácticas para que los estudiantes logren un aprendizaje significativo y transformador.

En el apartado anterior, se habló de la diferencia entre didáctica general y didáctica especial. De acuerdo con Díaz (2001), la didáctica universitaria se debe entender como una didáctica especial, enfocada en el diseño de estrategias de enseñanza en el salón de clases, en relación con la significatividad de los aprendizajes de los futuros profesionales. Las estrategias de enseñanza promueven diferentes tipos de conocimientos y de aprendizaje. En este sentido, la ausencia de didáctica universitaria se puede asociar con bajo el rendimiento estudiantil, debido a la ausencia de métodos y de recursos actualizados. La enseñanza requiere de la conceptualización e interpretación de las teorías que la fundamentan porque son la base del desarrollo integral para los estudiantes universitarios.

Según Zabalza-Beraza (2007; 2011), hablar de didáctica no es solamente hablar de pedagogía o de pedagogos, sino de docencia y docentes. Esto porque todo docente es didacta y desarrolla la labor didáctica. La docencia es el trabajo que el profesor universitario realiza en la formación de los estudiantes en el salón de clases y en los laboratorios. Al enseñar, el profesor debe poseer las competencias necesarias para realizar la tarea con eficacia. Los profesores pueden ocupar roles distintos, como el de investigadores o de administrativos, pero existe una identidad profesional que unifica la función y el desempeño profesional: ser docente.

El profesor principiante tiene una serie de limitantes-los temores y dudas propios del ejercicio, la poca o nula competencia científica, investigadora y didáctica, la inseguridad, la inexperiencia, entre otros-que provocan en él una incertidumbre constante. El profesor principiante en el proceso de inserción profesional atraviesa por un periodo de angustias, incertidumbres e inseguridades; independientemente del nivel educativo. La falta de formación inicial que caracteriza a los profesores de este nivel educativo los priva de un campo conceptual que fundamente su práctica docente. Y si, por alguna razón, el profesor principiante solicita ayuda de otros profesores que considera modelos, corre el riesgo de ser modelado por el estereotipo tradicional existente en la docencia universitaria: las clases magistrales (Mayor-Ruiz, 2010).

Algunos creen que la docencia es arte, arte de enseñar, no metodología. Otros consideran la docencia como arte, pero enfatizan en la importancia de la fundamentación científica de este arte pedagógico. Según Alanís-Huerta (2002), la docencia es el arte de enseñar un contenido específico, pero debe prepararse y planificarse, para que intervenga el elemento sistemático y la información científica. La docencia como arte de enseñar requiere de investigación científica.

Sin embargo, hay quienes no consideran la docencia como arte, sino como un proceso sistemático, científico. Si la docencia depende del arte, de la voluntad o pericia de cada profesor, el espacio para un saber sistemático es limitado. Entonces, la didáctica como disciplina poco tendría que aportar al conocimiento de las ciencias de la educación (Zabalza-Beraza, 2007). Por tanto, no puede ser una intuición artística, sino un planteamiento objetivo bien estructurado a través de procedimientos metodológicamente replicables.

La docencia se debe basar en una fundamentación didáctica estructurada y epistemológicamente diseñada. La experiencia medida en años laborales no es un argumento válido para fundamentar la actividad docente; pues no es equiparable a conocimientos de calidad. Entonces, la experiencia es la huella en la memoria que queda gracias a la actividad rutinaria a la que se ha estado involucrado voluntaria o involuntariamente. Por tanto, la realización rutinaria de una actividad durante años no significa la presencia de un aprendizaje nuevo, sino la realización mecánica de esta actividad. Es por esto que "la experiencia acumulativa y medida en años de servicio, como indicador de habilidades y conocimientos es una falacia." (Alanís-Huerta, 2002, p. 21).

Antes de iniciar una carrera profesional en docencia universitaria y empezar a acumular experiencia 
medida en años de existencia y no experiencia en tiempo formativo- epistemológicamente sustentable- es necesaria la formación formal en docencia. Gros-Salvat y Romañá-Blay (2012) en su investigación sobre docencia universitaria, afirman que ningún profesor novato debería enfrentarse con una clase sin algún tipo de preparación o apoyo previo. Tiene que existir un proceso gradual comenzando con actividades que supongan una menor responsabilidad y permitan la formación tanto de contenidos como de estrategias docentes.

¿Tiene importancia la docencia en el funcionamiento de una universidad? al respecto, Zabalza-Beraza (2007) tiene una opinión bastante aceptable. En el proceso de elección a rector, los candidatos proclaman esta importancia. Parece que la docencia constituye el elemento central, la sustancia del quehacer universitario. Sin embargo, la cotidianidad de las instituciones universitarias muestra la realidad de cómo se gestionan las prioridades, la forma cómo se incentiva al profesorado universitario y la manera en cómo este construye su identidad profesional. En conclusión, la docencia universitaria es un asunto marginal en el funcionamiento institucional y en el quehacer de muchos profesores.

En el contexto de la globalización y del avance de la ciencia y de la tecnología, la educación universitaria requiere un cambio de actitud importante en los docentes y en las políticas de las instituciones de educación superior. Los métodos de enseñanza no pueden continuar difundiéndose a través del docente de aula, labor para la cual cada día son menos competentes. Los docentes universitarios deben ampliar el horizonte didáctico y ser receptivos a los cambios en el campo de la tecnología educativa. No se trata de combatir el proceso de globalización, sino tratar de encausarlo para que favorezca la educación universitaria (Roldán, 2004).

\section{¿LO ESTAMOS HACIENDO BIEN? UNA REFLEXIÓN SOBRE LA PRÁCTICA DOCENTE}

Con frecuencia afirmamos que es importante el pensamiento crítico en nuestros estudiantes. Pero, ¿qué estrategia didáctica estamos implementando para lograrlo? Consideramos que dar a estudiantes cantidades abrumadoras de lectura garantiza ese anhelado pensamiento crítico, sin tomar en cuenta si la comprensión lectora en nuestros estudiantes universitarios es óptima. Un estudiante no adquiere pensamiento crítico por leer algunos textos políticos, científicos, ideológicos o filosóficos. El pensamiento crítico está inmerso en un proceso que requiere el desarrollo de habilidades metacognitivas, y para desplegar esa herramienta cognitiva en el estudiante, se tiene que trabajar en estrategias didácticas.

Quisiera ilustrar este apartado con una pequeña y breve anécdota. Cuando fui estudiante de grado en Filosofía (Bachillerato y Licenciatura) conocí un profesor que desarrollaba la clase de una forma tal, que opacaba la mayor parte del tiempo hablando y exaltando su forma correcta e incuestionable de interpretar un texto filosófico. Muchos años después, decidí realizar un posgrado. Lo tuve nuevamente como profesor... repitiendo el mismo esquema pedagógico. Esta vez, lo cuestioné mucho, tanto por la interpretación rígida y carente de sentido que hacía del texto filosófico, como por el procedimiento pedagógico que implementaba. Su respuesta fue: "Tengo más de veinte años de experiencia docente". Ergo, veinte años de arrastrar el mismo error pedagógico. A esto, lamentablemente, muchos Ilaman experiencia docente. Por cierto, se trataba del mismo texto filosófico que yo había estudiado cuando era un mancebo universitario, solo que ahora en un posgrado. Esto es muy frecuente en la docencia universitaria. Las zonas de confort pedagógico son elementos que se deben de tomar en cuenta como indicadores de errores didácticos en la enseñanza.

El recurso didáctico fundamental de muchos docentes es la clase magistral. Entiéndase por clase magistral, aquella en la cual el docente lo único que hace es hablar y hablar y hablar y hablar...y hablar. De esta forma, desarrolla una lección entera. Algunas lecciones se pueden prolongar hasta por tres horas continuas. Las clases magistrales semejan más el deseo narcisista de un profesor de demostrar su erudición empolvorada, que la disposición comprometida hacia el aprendizaje de los estudiantes. 
La docencia, en todos sus niveles, requiere de la implementación de estrategias didácticas si se quiere lograr un aprendizaje transformador en los estudiantes. Por eso, el docente de aula tiene que salir a investigar en el laboratorio de las ideas didácticas. La investigación didáctica permite la innovación educativa, la cual mejora la enseñanza en los docentes y el aprendizaje en los estudiantes.

Es importante tomar en cuenta que la investigación didáctica puede hacerse tanto a nivel documental como experimental; pues, la innovación didácticaal reposar en un procedimiento básico de tres fases: de planeación, de implementación y de evaluación—puede aplicarse a ambas. Desde un punto de vista de la investigación documental, se puede investigar sobre una estrategia en particular $y$, una vez que se adquiere un referente teórico sobre la estrategia, se planifica la sesión o sesiones de trabajo en el aula, se implementa en el salón de clases, y se evalúan los resultados posteriormente. Lo mismo aplica si nos decidimos por la experimentación didáctica: planificamos la estrategia, implementamos la estrategia y evaluamos la estrategia didáctica con los resultados obtenidos.

Es importante acotar que existen muchos procedimientos metodológicos aplicados a la investigación educativa. Si bien es cierto que la actuación directa del profesorado es un recurso, este procedimiento debe contar con un marco metodológico bien delimitado a fin de poder medir resultados, ya sea de forma cualitativa o cuantitativa. Para este cometido, existe abundante literatura sobre metodología de investigación aplicada a la educación.

Las investigaciones educativas pueden producir modelos didácticos de mucha utilidad práctica en el salón de clases. Un modelo didáctico tiene el doble propósito de facilitar la enseñanza en los docentes y el aprendizaje en los estudiantes. Lo más importante es que adaptar un modelo didáctico, puede sacarnos de la zona de confort pedagógica que caracteriza a muchos docentes universitarios.

En el contexto universitario, la investigación didáctica es fundamental para enfrentar los retos de aprendizaje del estudiante actual, inmerso en las tecnologías de la información. La docencia universitaria requiere de formación previa, de formación continua. Pero, sobre todo, requiere de investigación constante en el campo de la didáctica. Investigar en didáctica es reconocer el hecho de que pararse en frente de un grupo de estudiantes y hablarles magistralmente por horas no conlleva necesariamente al aprendizaje de contenidos. Por tanto, la innovación didáctica persigue mejorar la experiencia de la enseñanza y del aprendizaje.

No obstante, enfrentamos un reto en la docencia universitaria, el cual se compone de varias aristas. La docencia universitaria cuenta con poca identidad profesional. Y no se trata de identidad como profesor universitario; sino, más bien, de la identidad con la docencia como disciplina. El profesor universitario se identifica con su profesión, no con la profesión docente. En este sentido, la capacitación docente no se percibe como tal, sino como pérdida de tiempo, o bien, como un instrumento para ganar puntos para escalar de categoría profesional. Además, la formación en docencia universitaria no es un requisito curricular para aspirar a un puesto de profesor universitario.

Las unidades académicas solicitan experiencia docente como parte de los perfiles profesionales, previo a la contratación. Sin embargo, surge el problema de la calidad en la docencia universitaria que confunde la noción misma de experiencia docente. La experiencia acumulada y medida en años de actividad no necesariamente es un indicador de habilidades y de conocimientos didácticos en el campo de la docencia. Es decir, los años acumulados en el ejercicio docente no significan docencia de calidad para los estudiantes.

Por tanto, se puede contratar a un profesor universitario con cinco o más años de experiencia, pero esto no significa que se trata de experiencia en docencia universitaria, propiamente hablando. La experiencia en docencia universitaria implica investigación e innovación aplicada al mejoramiento de los procesos de enseñanza y aprendizaje, y no una rutina pedagógica basada en un discurso magistral monótono o en la presentación y lectura en voz alta de diapositivas 
de PowerPoint, rutinas que resultan aburridas para los estudiantes y no conducen a la motivación por el aprendizaje.

La docencia requiere experiencia, no cabe duda. Pero la docencia universitaria requiere de experiencia epistemológica y formativa, no solamente experiencia existencial. Esto porque no existe forma de someter a crítica epistemológica la propia experiencia, pues siempre se apelará a ella como criterio de verdad e, incluso, de autoridad. Existe un imaginario negativo de creer que los años en la actividad docente legitiman de autoridad pedagógica, lo cual es totalmente falaz. Un docente puede tener muchos años de dedicarse a la actividad docente, pero esto no garantiza que esta experiencia sea didácticamente transformadora para el aprendizaje de los estudiantes. En otras palabras, un docente puede tener años de experiencia cometiendo el mismo error pedagógico: la ausencia de estrategias didácticas para garantizar un aprendizaje transformador en los estudiantes.

La docencia universitaria no puede darse al margen de la investigación didáctica. Tampoco se puede equiparar la docencia con formación profesional. Un docente universitario requiere contar, necesariamente, con formación universitaria en un campo determinado; pero, también requiere contar con formación en docencia. Pues la actividad que desarrolla en el salón de clases es una actividad educativa, no técnica profesional. Su ejercicio técnico profesional lo puede ejercer en su negocio particular; puesto que, una vez que ingresa al salón de clases, deja de tener clientes y comienza a tener alumnos. Por tanto, debe sustituir el lenguaje técnico profesional por el lenguaje pedagógico. La formación profesional base es imprescindible, pero la formación en docencia universitaria es el complemento fundamental para desplegar ese conjunto de habilidades formativas, cuyo beneficiario último es el estudiante.

Por tal motivo, es inaceptable la tesis de ZabalzaBeraza (2007) de considerar que todos los profesores universitarios son didactas y realizan una labor didáctica. Considerar que todos los profesores universitarios son didactas contiene dos supuestos falaces: que la didáctica está implícita en la actividad docen- te o que la actividad docente implica la actividad didáctica como conocimiento. Ambos supuestos son, a todas luces, falsos. La didáctica no siempre es parte del ejercicio docente, ni el ejercicio docente se hace acompañar de estrategias didácticas para garantizar un mejor aprendizaje. Un profesor se hace didacta al estudiar y aplicar conocimientos didácticos o al investigar sobre didáctica.

Entonces, la actividad docente no implica necesariamente la presencia de actividad didáctica. Ya sea que se trate del inicio de la actividad docente en los primeros años de carrera profesional o de una actividad de años de experiencia, la didáctica no está necesariamente presente. $Y$ la razón es práctica: para ser docente universitario no se necesita ser graduado en docencia universitaria, al menos en el contexto costarricense. Es suficiente con ser una profesional en cualquier área de conocimiento y contar con la oportunidad de involucrarse en la actividad docente.

La docencia implica no solo vocación, sino formación. La vocación es un requisito indispensable para el ejercicio de cualquier profesión, pero no es suficiente. La actualización de conocimientos, la investigación, la práctica profesional, son fundamentales para el crecimiento. La formación e investigación permiten el crecimiento profesional en el campo de la docencia y en cualquier otro campo de conocimiento. La experiencia, por el simple hecho de medirse en años laborales, no garantiza necesariamente progreso epistemológico; pues, puede arrastrar un error por años, y la investigación puede evidenciar un error en uno pocos meses. La docencia bien sustentada es fundamental para el éxito de los estudiantes y el buen funcionamiento de una universidad.

\section{CONCLUSIONES}

Las consideraciones finales de este trabajo consisten en ofrecer una síntesis de los principales aportes teóricos y algunas acotaciones reflexivas sobre el tema tratado. Primero, se expone la síntesis del contenido teórico, seguido de algunas acotaciones reflexivas.

La importancia de la didáctica en los procesos de enseñanza y aprendizaje es fundamental. Para tal fin, la investigación didáctica es central; debido a 
que permite la innovación educativa. Dentro de sus múltiples objetivos, persigue la innovación de la docencia y la mejora de los aprendizajes de los estudiantes. Un proyecto de innovación didáctica es una estrategia que permite la planificación y la actuación del profesional de la educación. Es decir, es un proceso de indagación y solución que contempla tres fases elementales: planeación, implementación y evaluación.

La investigación educativa puede producir algún modelo didáctico. Todo modelo didáctico debe contar con los recursos necesarios que permita ponerlo en práctica, así como, con un marco de evaluación o valoración de resultados.

La evaluación de un modelo didáctico es muy importante, si se toma en cuenta que su objetivo es intervenir en el proceso pedagógico para optimizar la adquisición de competencias en los alumnos y facilitar la tarea del profesor. Además, no se puede perder la perspectiva de que la evaluación tiene que convertirse en una actividad de conocimiento y la corrección en un acto de aprendizaje.

En un proceso de planificación didáctica debe considerarse los cambios que se producen en las edades de los estudiantes para tratar de controlar, focalizar, dirigir y dinamizar el proceso de enseñanza-aprendizaje. Es importante que el planeamiento didáctico esté basado en un paradigma educativo y en un modelo pedagógico como referente teórico en el cual basar la práctica educativa

La estrategia se aplica a muchos campos, desde el militar hasta el administrativo. La educación no es ajena a la aplicación de estrategias; pues, son herramientas del pensamiento que buscan alcanzar objetivos o metas de aprendizaje. En el campo de la educación, la estrategia se comprende como un conjunto de instrumentos o procedimientos que se implementan para alcanzar competencias de aprendizaje en los estudiantes. Su prueba final de validez se da en la práctica, en la acción.

La docencia universitaria no puede enfocarse únicamente en la enseñanza, sino que debe contemplar la investigación didáctica. La investigación educativa tiene el propósito de mejorar la calidad de los procesos de enseñanza-aprendizaje y la calidad profesional de los docentes.

En esta línea, la docencia universitaria mejora a través de la investigación didáctica. Por lo cual, se pueden considerar dos alternativas metodológicas básicas: la actuación directa del profesorado en el aula y los procesos de observación, de reflexión y de análisis sobre la propia práctica docente y creencias pedagógicas de los profesores, que pueden influir en el aprendizaje de los estudiantes.

La identidad profesional como docente universitario y la aplicación de estrategias didácticas por parte del docente en la enseñanza superior, son temas críticos. Se considera que la enseñanza universitaria cuenta con poca identidad profesional, pese a la condición de profesor universitario. Pues este no se prepara realmente para ser docente universitario, $y$, por ende, esta carencia formativa, se traduce en carencia de didáctica universitaria que pueden afectar el rendimiento de los estudiantes debido a la ausencia de métodos y recursos actualizados. No todos los profesores universitarios son didactas ni realizan una labor didáctica.

Luego de esta exposición sobre los principales puntos desarrollados en el trabajo, se exponen algunas acotaciones reflexivas, de acuerdo con el contenido y el apartado final.

No es lo mismo tener carencias pedagógicas y limitaciones didácticas por falta de experiencia en la labor docente, que tener estas carencias y limitaciones por falta de actualización profesional. La inversión personal en bibliografía educativa puede contribuir con la actualización de conocimientos pedagógicos y didácticos. Pero no se puede vivir actualizado, sino se tiene el hábito de invertir en formación personal.

Mejorar la calidad de la educación requiere, es cierto, de todo un proceso administrativo que puede demandar inversión económica. No obstante, también es cierto que la inversión individual contribuye con este propósito. 
El papel de la didáctica en los procesos de enseñanza y aprendizaje universitarios

La investigación didáctica puede ofrecer insumos teóricos importantes para orientar la práctica docente. Este tipo de investigación puede ser experimental o teórica. El producto de estos proyectos de investigación, se concreta en herramientas para la enseñanza, como las estrategias didácticas, que se pueden aplicar directamente en el salón de clases y ofrecen la posibilidad de cambiar favorablemente la experiencia pedagógica.

Los profesionales en educación deben asumir la investigación como parte de la labor docente. Investigar en educación significa innovar y actualizar conocimientos, y es una ventana a la reflexión pedagógica cotidiana.

\section{REFERENCIAS BIBLIOGRÁFICAS}

Alanís-Huerta, A. (2002). El saber hacer en la profesión docente. México: Trillas.

Barraza-Macías, A. (2015). Proyectos de innovación didáctica para la mejora de la práctica docente. México: Instituto Universitario Anglo Español. Recuperado de http://iunaes.mx/ wp-content/uploads/2015/12/PROYECTOS-DEINNOVACI\%C3\%93N-DIDACTICA-PARA-LAMEJORA-DE-LA-PR\%C3\%81CTICA-DOCENTE. pdf

Cantú-Lozano, E. (2016). Enséñame a aprender. México: Trillas.

Cervera, D (Coord.). (2010). Formación del profesorado. Educación secundaria. Madrid: Ministerio de Educación, Cultura y Deportes.

Chadwick, C. (1999). La psicología del aprendizaje desde el enfoque constructivista. Revista Latinoamericana de Psicología, 31(3), 463-475. Recuperado de http://www.redalyc.org/pdf/805/80531303. pdf

Díaz, D. (2001). La didáctica universitaria: una alternativa para transformar la enseñanza. Acción Pedagógica, 10 (1-2), 64-72

Escribano-González, A. (2004). Aprender a enseñar: fundamentos de didáctica general. Cuenca: Edi- ciones de la Universidad de Castilla-La Mancha. Recuperado de https://books.google.co.cr

García, J. (s.f. de 2015). El padre de la didáctica: Juan Amos Comenio [Blog de Universidad Justo Sierra]. Recuperado de http://www.universidad-justosierra. edu.mx/wordpress/el-padre-de-la-didactica-juanamos-comenio/

Gros-Salvat, B. y Romañá-Blay, T. (2012). Ser profesor: palabras sobre la docencia universitaria. Barcelona: Octaedro

Hernández-Sampieri, R. (2014). Metodología de la investigación. 6 ed. México: McGRAW-HILL

Mayor-Ruiz, C. (2010). El desafío de los profesores principiantes universitarios ante su formación. En C. Marcelo (Coord.), El profesorado principiante: inserción a la docencia. Barcelona: Octaedro

Medina, A. \& Medina, M. (2014). La investigación como base del conocimiento didáctico y de la innovación en la enseñanza. En A. Medina-Rivilla., A. De la Herrán-Gascón \& M. Domínguez-Garrido. Fronteras en la investigación de la didáctica. Madrid: UNED. Recuperado de http://www.ebrary. com

Montealegre-García, C. (2016). Estrategias para la enseñanza y el aprendizaje de las ciencias. Ibagué: Universidad de Ibagué. Recuperado de http:// ebookcentral.proquest.com

Ortega-Alfaro, R. (2017). Estrategias didácticas y evaluación de competencias. México: Trillas

Pimienta-Prieto, J. (2012). Estrategias de enseñanzaaprendizaje. Docencia universitaria basada en competencias. México: Pearson

Prieto-Navarro, L (2007). Autoeficacia del profesor universitario: eficacia percibida y práctica docente. Madrid: Narcea Ediciones.

Rodríguez-Gómez, D. \& Valldeoriola-Roquet, J. (2009). Metodología de la investigación. Barcelona: UOC 
Rojas-Vargas, A. (2016). Planeamiento del proceso de enseñanza-aprendizaje. San José, C. R: EUNED

Roldán, M. (2004). Globalización, educación costarricense y didáctica universitaria hoy. Revista Reflexiones 83 (2), 37-49

Sánchez-Romero, C. (2013). Aplicación de estrategias didácticas en contextos desfavorecidos. Madrid: UNED. Recuperado de https://ebookcentral. proquest.com

Sebastiá-Alcaraz, R. (2012). Investigación e innovación en Didáctica de las Ciencias Sociales: Historia. Alicante: Universidad de Alicante. Departamento de Didáctica General y Didácticas Específicas. Recuperado de http://hdl.handle.net/10045/36975

Torres-Maldonado, H. \& Girón, D. (2009). Didáctica general. San José: Coordinación Educativa y Cultural Centroamericana. Recuperado de http:// unpan1.un.org/intradoc/groups/public/documents/ icap/unpan039746.pdf

Tovstiga, G. (2012). Estrategia en la práctica: la guía profesional para el pensamiento estratégico. Buenos Aires: Granica. Recuperado de https://books. google.co.cr

Zabalza-Beraza, M. (2005). Competencias docentes del profesorado universitario: calidad y desarrollo profesional. Madrid: Narcea Ediciones

Zabalza-Beraza, M. (2007). Didáctica universitaria. Bordón 59(2-3), 489-509. Recuperado de https:// dialnet.unirioja.es/descarga/articulo/2553099.pdf.

Zabalza-Beraza, M. (2011). Nuevos enfoques para la didáctica universitaria actual. Perspectiva, Florianópolis, 29(2), 387-416. Recuperado de https:// periodicos.ufsc.br/index.php/perspectiva/article/ download/2175-795X.2011v29n2p387/22209 